\title{
Noncirrhotic portal fibrosis: a rare cause of end-stage liver disease requiring liver transplantation
}

\author{
Sunil Taneja $\cdot$ Yogesh Chawla $\cdot$ Radha K. Dhiman
}

Received: 16 August 2011/Accepted: 18 August 2011/Published online: 3 September 2011

(C) Asian Pacific Association for the Study of the Liver 2011

Non-cirrhotic portal fibrosis (NCPF) is a disease of uncertain etiology characterized by periportal fibrosis and involvement of small and medium branches of the portal vein, resulting in the development of portal hypertension [1]. This has been reported from different parts of the world under a variety of names, such as idiopathic portal hypertension, hepatoportal sclerosis, and noncirrhotic intrahepatic portal hypertension, etc. In India, NCPF formed an important group of patients with portal hypertension, and accounted for $15-30 \%$ of all such patients in early nineties [2]. However, its incidence has considerably decreased over time and it is now believed to be a vanishing disease in the subcontinent [1]. Improvement in sanitary conditions and decrease in bacterial infections has lead to a decline in the incidence of the disease. Similar drop in the incidence of new cases has been reported from Japan and other countries $[1,3]$.

How NCPF differs from cirrhosis of liver has generated immense interest over the years, although at times it may not be possible to differentiate NCPF from Child A cirrhosis of liver. Majority of these patients present with features of portal hypertension and well-tolerated variceal bleeding and have good long-term prognosis $[1,4]$. A long-term followup study of 59 patients with noncirrhotic intrahepatic portal hypertension in one hospital over 30-year period showed a 5 -year survival of $90 \%$ and a 30 -year survival of 55\% [5]. However, a small proportion of patients do behave like endstage liver disease and develop features of decompensation

S. Taneja $\cdot$ Y. Chawla $\cdot$ R. K. Dhiman $(\bowtie)$

Department of Hepatology,

Postgraduate Institute of Medical

Education and Research, Chandigarh 160012, India

e-mail: rkpsdhiman@hotmail.com in the form of ascites, jaundice and hepatic encephalopathy. This subgroup of NCPF with bad liver functions has been recognized and constitutes $<10 \%$ of the patients, who have mild jaundice, mild ascites and low serum albumin $[4,6]$. Nodular transformation of the liver with extensive subhepatic and portal fibrosis has been reported in these patients and the condition constitutes a late manifestation of NCPF. Incomplete septal cirrhosis and nodular regenerative hyperplasia may also be associated with NCPF or idiopathic portal hypertension and may be the late manifestation of this disorder [7-10]. These severe histological changes and abnormal liver function have been infrequently reported and require longer follow-up studies to look into this aspect.

There are no large studies that have looked at the subgroup of patients who undergo decompensation; however, in some patients with $\mathrm{NCPH}$, the complications of portal hypertension are sufficiently severe to warrant liver transplantation [7, 11-16]. The study in this issue of Hepatology International shows that small fraction of patients $(\sim 5 \%)$ do develop ascites, jaundice, and hepatic encephalopathy making it difficult to differentiate it from cirrhosis of liver [17]. Another interesting observation of this study is the overlap NCPF group which has features of both NCPF and cirrhosis and was not reported earlier. This was seen in ten patients in which cause of chronic liver disease was hepatitis $\mathrm{C}$ in five, hepatitis $\mathrm{B}$ in one and nonalcoholic fatty liver disease (NAFLD) in four patients. All ten patients in this group had characteristic portal vein changes. This may be in continuum with the spectrum of NCPF not defined earlier due to absence of serological markers of hepatitis B and $\mathrm{C}$ and recent identification of NAFLD as a cause of significant liver disease; although nodular regenerative hyperplasia and incomplete septal cirrhosis have been reported earlier. The existence of two diseases in a single 
patient may portend a poor prognosis as progression to end-stage liver disease may be faster.

Pathologically, NCPF is characterized by small portal vein obliteration, aberrant vasculature, portal tract fibrosis which is rounded or streak and absence of significant hepatocellular injury and formation of regenerative nodules characteristic of cirrhosis [1]. This paper emphasizes the classical histopathological findings of NCPF with all ten patients showing obliterative portal venopathy and portal to portal fibrosis. Irregular coarse granularity and small nodules on the surface were seen in eight and seven patients respectively differentiating NCPF from cirrhosis of liver. In overlap NCPF the existence of two diseases has not been described earlier, this study gives insight into the probable different pathogenic mechanisms and coexistence of the two diseases with different pathological findings.

How does NCPF progresses to end-stage liver disease and liver failure is not known, although it has been postulated that as time progresses these patients develop progressive atrophy of liver owing to reduced blood supply to the periphery and subsequent manifestations of liver dysfunction [1].

This study has clearly demonstrated that the diagnosis of NCPF can easily be missed prior to the liver transplant and has had a pre-transplant diagnosis of cirrhosis of unknown or some known etiology. How could a pretransplant diagnosis of NCPF be arrived? NCPF should be suspected in cases of chronic liver disease with negative etiological work up, relatively preserved liver functions and a low MELD score. In this study seven objective and two subjective parameters showed significant differences between pure NCPF and NAFLD control groups, however, as proposed by the authors need further prospective validation. Should all patients with suspected NCPF or cryptogenic cirrhosis posted for liver transplantation subjected to liver biopsy? The biopsy in NCPF may be patchy and inconclusive as seen from explants liver histopathology. The hemodynamic changes in NCPF also differ from cirrhotics and demonstrate markedly elevated intrasplenic and portal vein pressures while the hepatic venous pressure gradient may be normal or slightly elevated because the site of resistance is predominantly presinusoidal [1]. The routine use of hepatic venous pressure gradient for differentiation of NCPF from cirrhosis is still underutilized and may prove to be a useful tool to differentiate sinusoidal from non-cirrhotic portal hypertension in this setting.

The indication for liver transplantation in NCPF still remains to be defined as no definite guidelines exist. MELD which is the strongest predictor of prognosis and post-transplant outcome and may not be applicable for organ allocation in NCPF due to fairly well preserved liver functions as seen in this study with all patients having low MELD scores and preserved liver functions. In this situation, the indication for transplant is primarily based on complications of portal hypertension. The use of a new terminology of overlap NCPF as an indication for liver transplantation in this paper is clinically not relevant since it is a histopathological diagnosis and explant liver histology with careful examination is required.

The post-transplant outcome of the patients with NCPF is good and not different from other end-stage liver disease patients of different etiologies [18]. Recurrence of disease post-transplantation would not be an intriguing issue since it is a slowly progressive disease.

In summary, this study highlights NCPF as a rare cause of end-stage liver disease requiring liver transplantation. It does raise the interesting issue of chronic liver disease of other etiology, may supervene on preexistent NCPF and might have faster progression to end-stage liver disease. The diagnosis of NCPF can be easily missed prior to transplant and may become apparent only when explants liver is carefully examined. NCPF is a great mimicker of cirrhosis clinically and radiologically.

\section{References}

1. Sarin SK, Kumar A, Chawla YK, Baijal SS, Dhiman RK, Jafri W, et al. Noncirrhotic portal fibrosis/idiopathic portal hypertension: APASL recommendations for diagnosis and treatment. Hepatol Int 2007;1:398-413

2. Dilawari JB, Bambery P, Chawla Y, Kaur U, Bhusnurmath SR, Malhotra HS, et al. Hepatic outflow obstruction (Budd-Chiari syndrome). Experience with 177 patients and a review of the literature. Medicine (Baltimore) 1994;73:21-36

3. Imai F, Kuga K, Komaba M, et al. Interim report on IPH survey. In Futagawa S, editor. 1992 Report of the Research Committee on Aberrant Portal Hemodynamics. Tokyo: Ministry of Health and Welfare; 1993. 107-110

4. Dhiman RK, Chawla YK, Vashista RK, Kakkar N, Dilawari JB, Puri P, et al. Noncirrhotic portal fibrosis (NCPF). A study of 151 cases and review of literature. J Gastroenterol Hepatol 2002;17: 6-16

5. Kingham JG, Levison DA, Stansfeld AG, Dawson AM. Noncirrhotic intrahepatic portal hypertension: a long term follow-up study. Q J Med 1981;50:259-268

6. Tandon BN, Nundy S, Nayak NC. Non-cirrhotic portal hypertension in Northern India. Clinical features and liver function tests. In Okuda K, Omata M, editors. Idiopathic Portal Hypertension. Tokyo: University of Tokyo Press; 1983. 377-386

7. Bernard PH, Le Bail B, Cransac M, Barcina MG, Carles J, Balabaud C, Bioulac-Sage P. Progression from idiopathic portal hypertension to incomplete septal cirrhosis with liver failure requiring liver transplantation. J Hepatol 1995;22:495-499

8. Baldus WP. Idiopathic portal hypertension: a histopathological study of 26 Japanese cases. Histopathology 1993;22:227-234

9. Sciot R, Staessen D, Van Damme B, Van Steenbergen W, Fevery J, De Groote J, Desmet VJ. Incomplete septal cirrhosis: histopathological aspects. Histopathology 1988;13:593-603

10. Krasinskas AM, Eghtesad B, Kamath PS, Demetris AJ, Abraham SC. Liver transplantation for severe intrahepatic noncirrhotic portal hypertension. Liver Transpl 2005;11:627-634 
11. Stromeyer FW, Ishak KG. Nodular transformation (nodular "regenerative" hyperplasia) of the liver. A clinicopathologic study of 30 cases. Hum Pathol 1981;12:60-71

12. Elariny HA, Mizrahi SS, Hayes DH, Boudreaux JP, Hussey JL, Farr GH Jr. Nodular regenerative hyperplasia: a controversial indication for orthotopic liver transplantation. Transpl Int 1994;7: 309-313

13. Radomski JS, Chojnacki KA, Moritz MJ, Rubin R, Armenti VT, Wilson GA, et al. Results of liver transplantation for nodular regenerative hyperplasia. Am Surg 2000;66:1067-1070

14. Loinaz C, Colina F, Musella M, Lopez-Rios F, Gomez R, Jimenez $\mathrm{C}$, et al. Orthotopic liver transplantation in 4 patients with portal hypertension and non-cirrhotic nodular liver. Hepatogastroenterology 1998;45:1787-1794

15. Dumortier J, Bizollon T, Scoazec JY, Chevallier M, Bancel B, Berger $\mathrm{F}$, et al. Orthotopic liver transplantation for idiopathic portal hypertension: indications and outcome. Scand J Gastroenterol 2001;36:417-422

16. Blanc JF, Bernard PH, Le Bail B, De Ledinghen V, Saric J, Baladaud C, et al. Vascular pathology of the portal vein distal branches: a rare cause of liver transplantation and a protean clinical presentation [in French]. Gastroenterol Clin Biol 2000; 24:667-670

17. Saigal S, Nayak NC, Jain D, Kumaran V, Mohanka R, Saraf N, et al. Noncirrhotic portal fibrosis related end stage liver disease in adults: evaluation from a study on living donor liver transplant recipients. Hepatol Int 2011;5

18. Jain A, Reyes J, Kashyap R, Dodson SF, Demetris AJ, Ruppert K, et al. Long-term survival after liver transplantation in 4, 000 consecutive patients at a single center. Ann Surg 2000;232: 490-500 